\title{
COMPARISON OF WAVELET SPECTRAL DENSITY AND RESCALED RANGE METHODS FOR HURST PARAMETER ESTIMATION
}

\section{JULIÁN R. CAMARGO L, MIGUEL A. ÁVILA A \& OSCAR D. FLÓREZ C}

Engineering Faculty, Universidad Distrital Francisco José de Caldas, Bogotá, Colombia

ABSTRACT
In this paper, two methods are presented for estimating the Hurst parameter using the Matlab computer tool. The analysis
is done using images in typical JPG format, which, given their size, allow for data traces of more than one million. With
the use of the two proposed methods, a comparison of the efficiency of the implemented algorithms is made in terms of
computational complexity and the robustness of the estimator.
KEYWORDS: Estimator, Hurst Parameter, Matlab, Rescaled Range \& Wavelet Spectral Density

Received: Jun 05, 2020; Accepted: Jun 25, 2020; Published: Oct 05, 2020; Paper Id.: IJMPERDJUN20201479

\section{INTRODUCTION}

The Hurst exponent takes its name from the engineer Harold Edwin Hurst (1880-1978), who dedicated his life to exploring the Nile River along with other rivers and conducting studies regarding water storage problems in the dams. Hurst discovered that, in contrast to other rivers, the water levels of the Nile over 800 years followed a selfsimilar pattern of variability, and consequently developed a standardized dimensionless measure to characterize that variability, calling it a rescaled range [1], [2].

Hurst's exponent estimation is applied in multiple systems covering from biophysics to computer networks. This method was initially designed for hydrological studies, but now fractal mathematics is used to estimate the Hurst exponent. This estimation generates a measure to understand if the studied data have a pure random trajectory or if they have different trends [2], [3].

\subsection{Estimation of Hurst's Exponent Using Spectral Density of the Wavelet Transform}

The Hurst exponent for a data set can be obtained by making use of the wavelet spectral density, which is often referred to as the scalogram. The wavelet spectral density graph is obtained from the wavelet power spectrum [4]. To calculate the standardized power for the j-octave, (1) is used.

$$
P_{j}=\frac{1}{2^{j}} \sum_{i=0}^{2^{j}-1} C_{i}^{2}
$$

The power is found with the sum of the squares of the wavelet coefficients (which corresponds to the forward wavelet transform) for the $j$-octave. A wavelet octave includes $2 j$ wavelet coefficients [5], [6]. The normalized power corresponds to the sum of the normalized squares divided by $2 \mathrm{j}$. In spectral analysis, the use of the normalized power is not always necessary, but in practice, to find the Hurst exponent, the normalized power is required.

Figure 1 presents the normalized power density graph for the 1024 data point wavelet transform that 
generates a Hurst exponent of 0.72 as a result. The Daubechies D4 wavelet was used for this, since the $\log 2$ of the number of wavelet coefficients corresponds to this number of wavelet octaves, it should be noted that for this case the $\mathrm{x}$-axis is on a logarithmic scale [6].

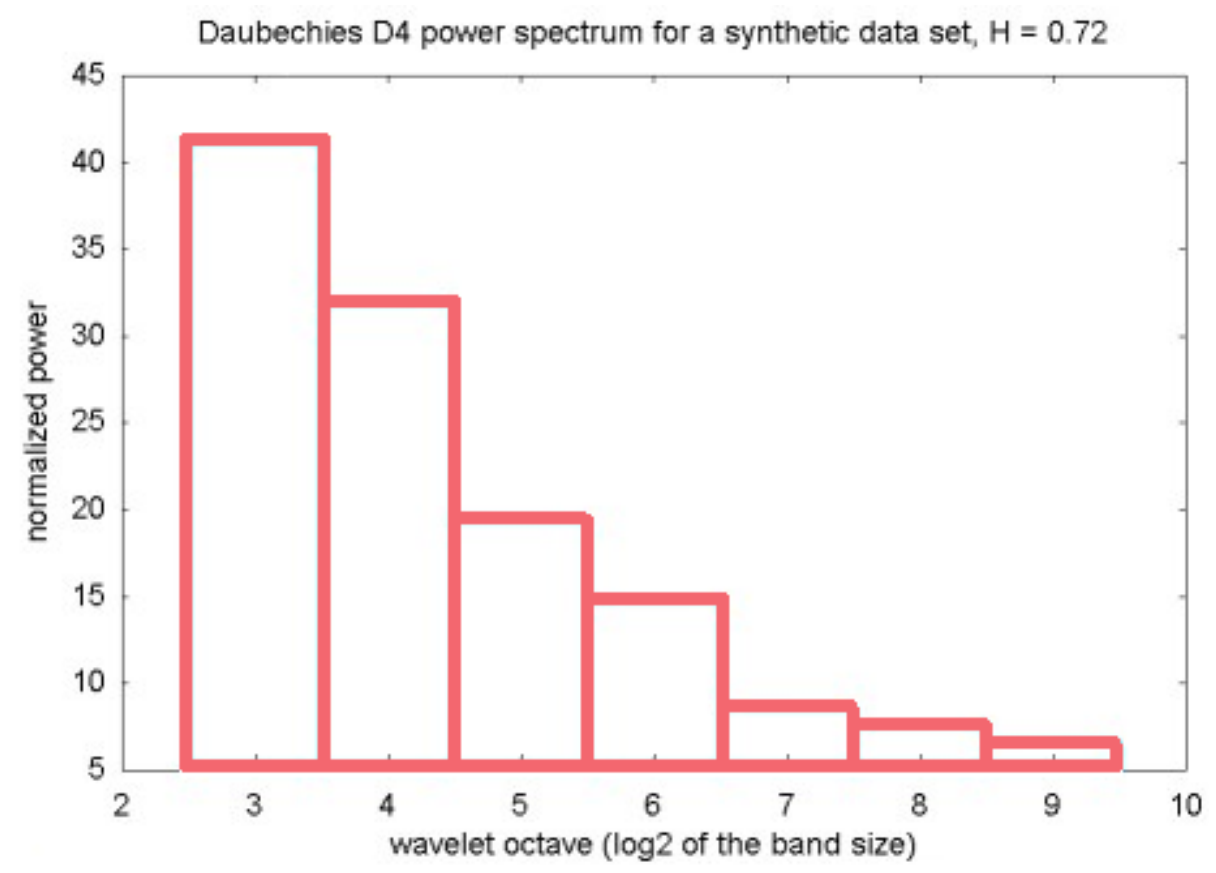

Figure 1: Standardized Power Density [6].

If a precise answer is desired, instead of an approximation or an idea of how a change in one thing generates a change in another thing, the statement that that thing is proportional to the other thing is not useful for many cases [6], [8]. The equation for calculating the exact value of Hurst's exponent using the slope of the line generated by linear regression using normalized spectral density is presented in (2).

$$
H=\left|\frac{m-1}{2}\right|
$$

\subsection{Rescaled Range Calculation Used to Estimate the Hurst Exponent}

The method developed by Hurst allows an "H" estimate to be made, and can be shown as follows:

The problem of designing a dam to regulate water flow, based on available records (time series), is addressed with an ideal dam model, in which the outlet flow is constant and equal to the average inlet, but considering that the dam never overflows and never empties [6], [9]. In this design it is necessary to know the measure of the variability of the water flow, and how the annual records are available. Figure 2 presents the problem described. 


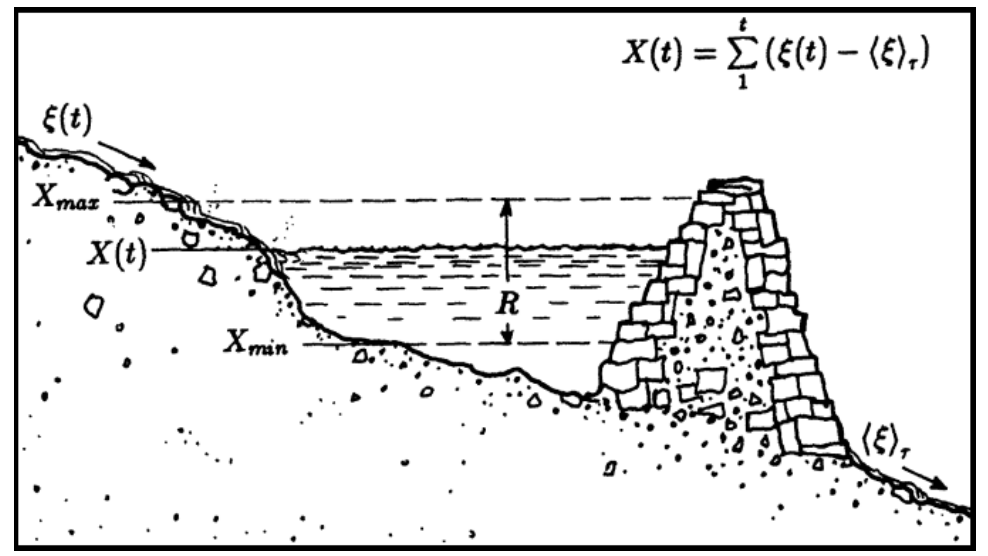

Figure 2: RiverNile [10].

Where,

- The variable $\xi(t)$ represents the entry of water into the reservoir.

- $\langle\xi\rangle_{\tau}$ represents the average water output in $\tau$ years.

- $\quad X(t)=-\sum_{1}^{t}\left[\xi(t)-\langle\xi\rangle_{\tau}\right]$ represents the water level, at year $\mathrm{t}$.

- Xmax represents the maximum accumulated level up to year $\mathrm{t}$.

- $\quad$ Xmin represents the minimum accumulated level up to year $\mathrm{t}$.

- $\mathrm{R}$ represents the range of variation between the maximum water level and the minimum level of the reservoir up to year t.

The time series $X(t)$, represents at a given moment $t$, the accumulated sum of the differences between the point value and the average value of water output, it can be considered that it is the amount of accumulated water by the value of which the time series deviates from its average until moment $t$, then if the maximum value is taken, and the minimum value, the value of the range $\mathrm{R}$ is obtained by difference, (3) represents the above [10], [11].

$$
R(t)=\left[\max \sum_{1}^{t} X(t)-[X(t)]-\sum_{1}^{t} X(t)-[X(t)]\right]
$$

In a way, this represents the variability of $X$, concerning its mean [10], [12]. Now, being $S(t)$, the standard deviation of $\mathrm{X}(\mathrm{t})$, we have (4).

$$
S(t)=\sqrt{\frac{1}{\tau} \sum_{t=1}^{\tau}(\zeta(t)-[\xi(t)])^{2}}
$$

Then, the measure of the range is normalized by dividing $\mathrm{R}$, by the value of $\mathrm{S}$ and the R/S measure is obtained, to which Hurst gave the name of the rescaled range because, in his investigations, he found that for many natural phenomena as it can be, the discharge of the rivers, the accumulation of sediments, the rings of the trees, and some statistical series, the empirical relation $\mathrm{R} / \mathrm{S} \sim(\mathrm{N} / 2) \mathrm{H}$ with $\mathrm{H}>0.5$, for big values of $\mathrm{N}$, describes quite well the observed phenomenon. 
Applying logarithms and their properties, this relationship can be written as shown in (5).

$$
\log \left[\frac{R}{S}\right] \approx H \log (N) \approx H \log (2)
$$

As you can see, if the points are represented as $\log (\mathrm{R} / \mathrm{S})$ against $\log (\mathrm{N})$, they will be adjusted to a straight line of slope $\mathrm{H}$, and an order to the origin equal to $\mathrm{H} \log (2)$. This analysis is called R/S statistic, or R/S graph.

Based on this, an estimator of the value of $\mathrm{H}$ can be developed, calculating different points of the rescaled range and then, using linear regression, calculating the value of the slope that represents the value of $H$ [13], [14].

\section{METHODOLOGY}

\subsection{Obtaining the Data Trace}

The data-trace was obtained from an image (photograph), in which the three base color matrices (Red, Green and Blue) were concatenated in an orderly fashion and placed in a vector of the total data. The code used to obtain the data-trace using the Matlab tool is shown in Figure 3.

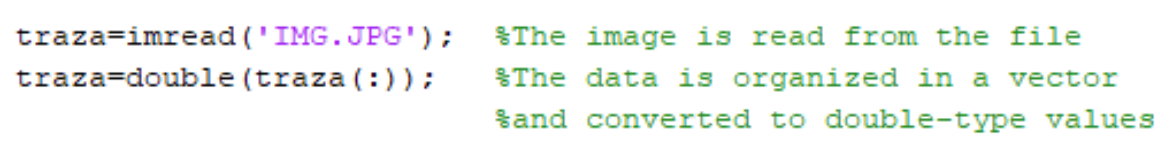

Figure 3: Code To Obtain The Data-Trace.

To visualize the data, the code shown in Figure 4 was generated.

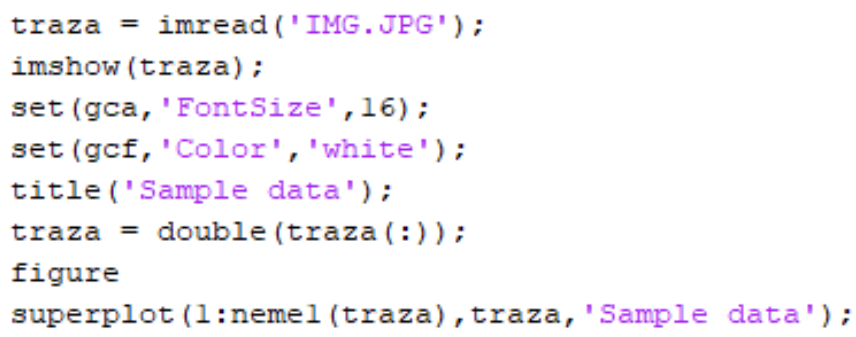

Figure 4: Code in Matlab to Display the Data.

Figure 5 shows one of the images used to perform the data analysis.

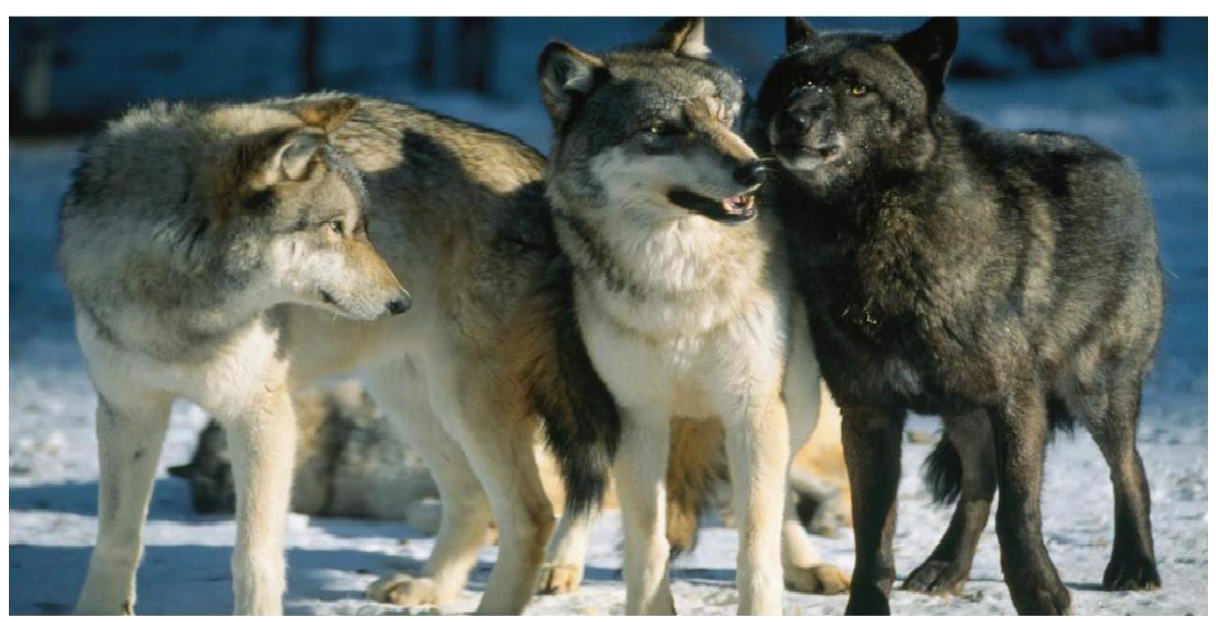

Figure 5: Image Analyzed in Matlab. 
Figure 6 shows the data-trace obtained for the previous image after running the algorithm in Matlab.

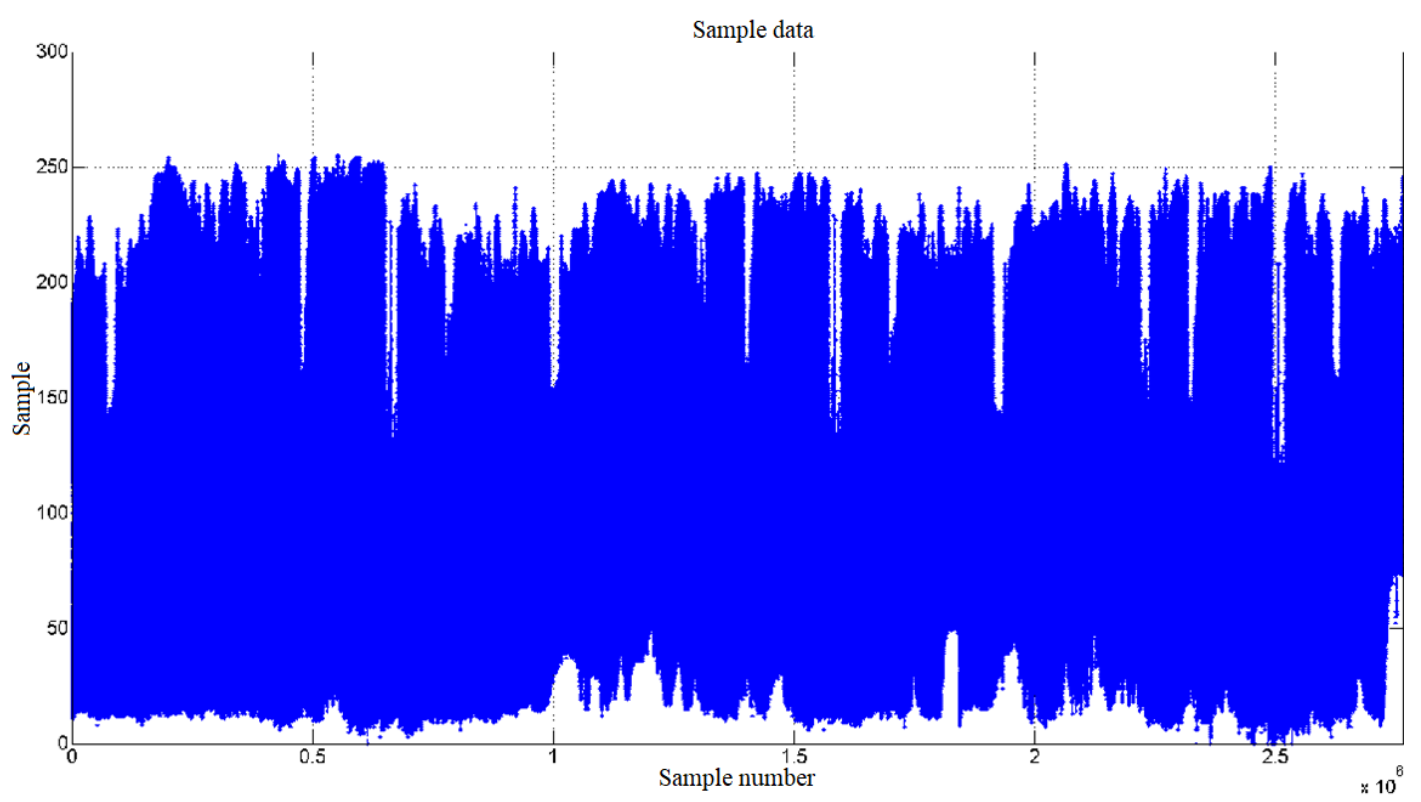

Figure 6: Data Trace.

\subsection{Calculation of the H-exponent Using Power Spectral Density of the Wavelet Transform}

To calculate the Hurst coefficient using the power spectral density, it is necessary to first calculate the Wavelet transformer coefficients. Matlab's Toolbox Wave contains the wavedec function that calculates these coefficients, as shown in figure 7.

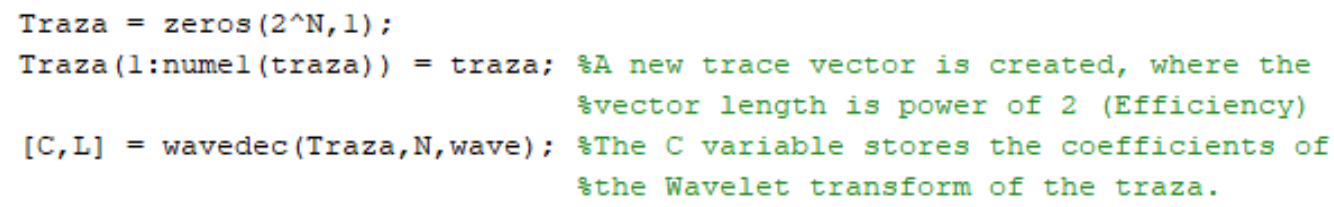

Figure 7: Using the Matlabwavedec Function.

Subsequently, it is required to calculate the standardized power coefficients using (1), to do so, the code shown in Figure 8 is generated.

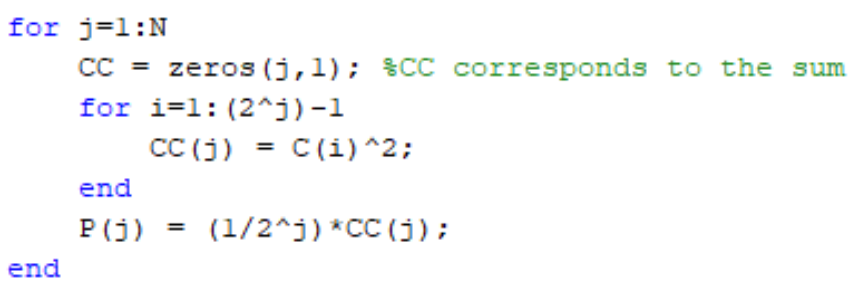

Figure 8: Calculation of the Standardized Power Coefficients.

Then the linear regression of the coefficients is performed after applying logarithm in base 10, this was achieved using the pendienteci function, as shown in figure 9.

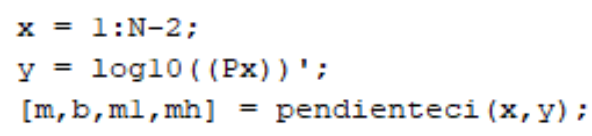

Figure 9: Linear Regression of the Coefficients. 
The calculation of Hurst's exponent is done using (2). Finally, to obtain the parameter and the graphs, what is shown in Figure 10 was done.

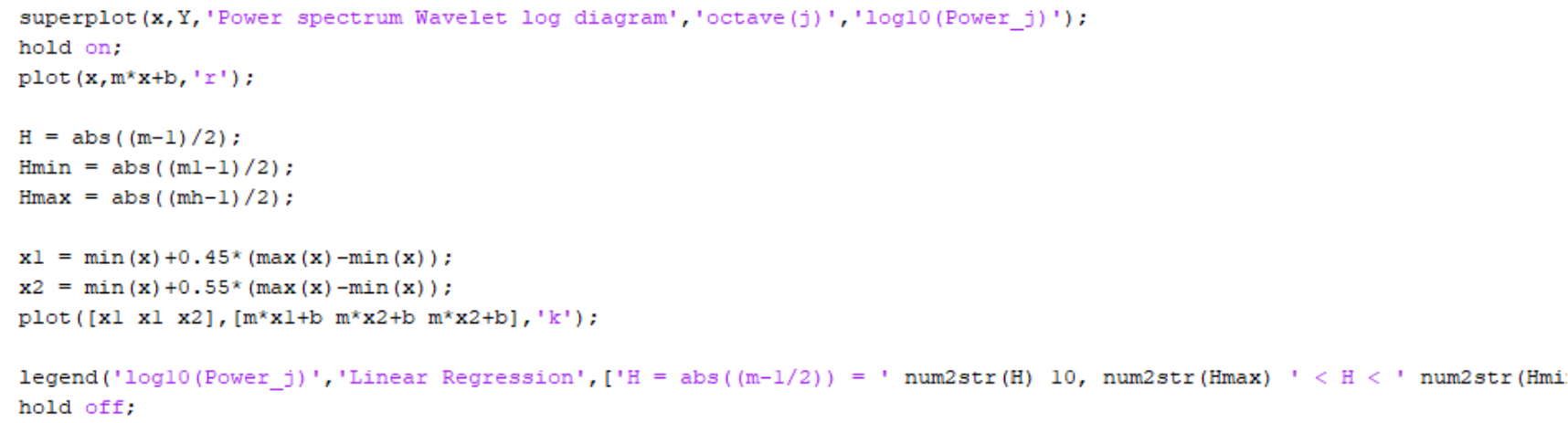

Figure 10: Final Calculation of Hurst's Exponent.

To test the developed function, all the code in Matlab shown in the previous steps is joined, obtaining the graph shown in figure 11 , giving as a result value of $\mathrm{H}=0.81829$.

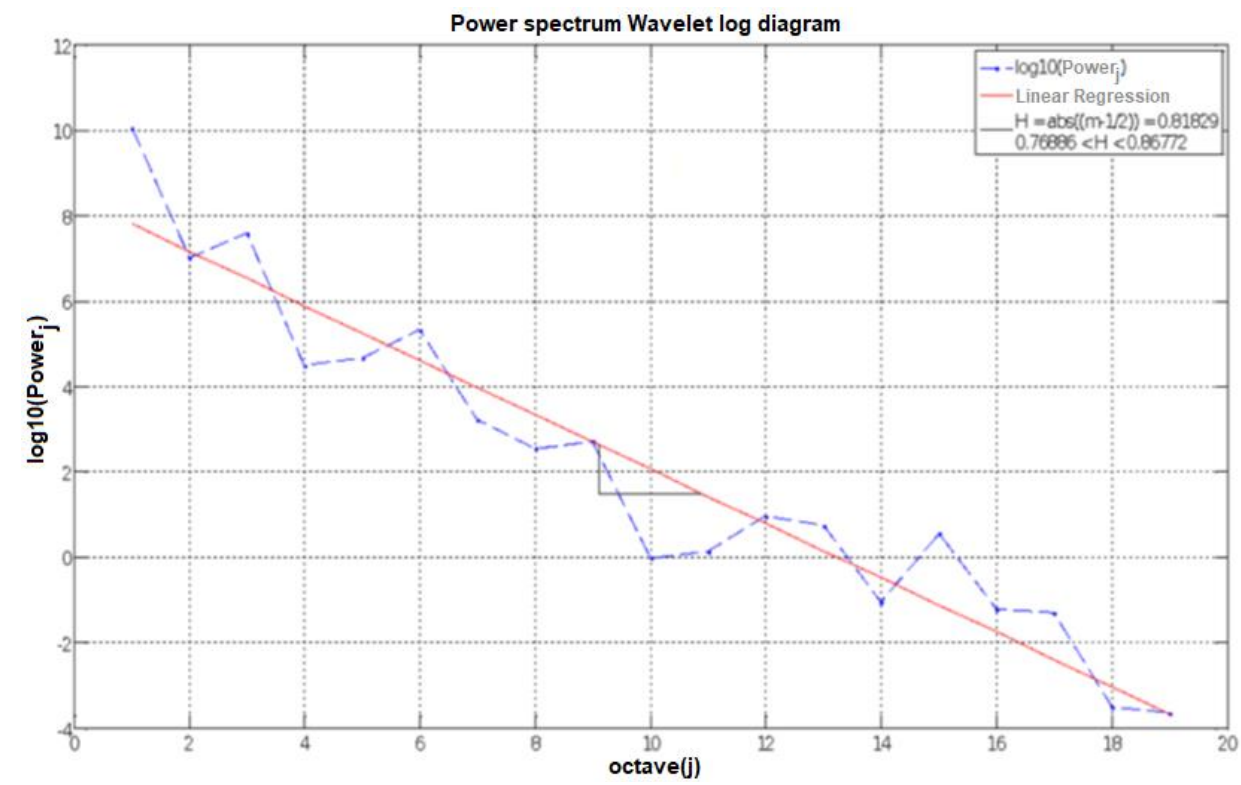

Figure 11: Result of Applying the HWavelet Function to the Data-Trace.

\subsection{Calculation of the H-exponent Using Rescaled Range (R/S)}

To perform the calculation of the Hurst coefficient using the rescaled range, it is necessary to follow the following process:

- $\quad$ From a vector with the data-trace, the studied process is sampled, for various sample sizes "N".

- The value of the rescaled $\mathrm{R} / \mathrm{S}$ range ratio is calculated for each sample.

- The logarithm of the rescaled range (R/S) and the logarithm of the number of samples (N) are calculated by plotting the points.

- The value of $\mathrm{H}$ is estimated by linear regression applied to the least-squares method.

- Finally, the R/S graph with the calculated points, the line of the regression and the number of samples taken for the calculations is delivered as an output. 
The process then starts with the Matlab code shown in figure 12 where each of the points that will be used to generate the linear regression is obtained.

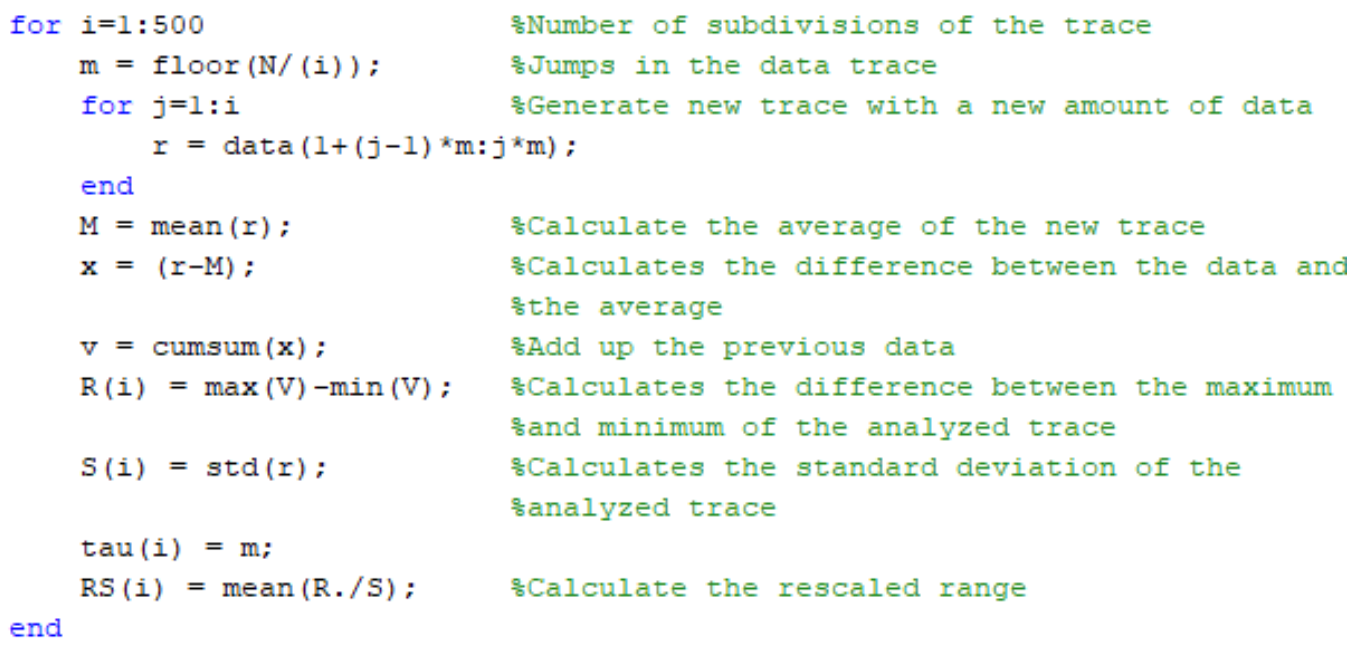

Figure 12: Algorithm to Generate the Points used in the Linear Regression.

With the points obtained, linear regression is generated, with the code shown in figure 13, with which the value of the parameter $\mathrm{H}$ is obtained, which corresponds directly to the slope of the line obtained.

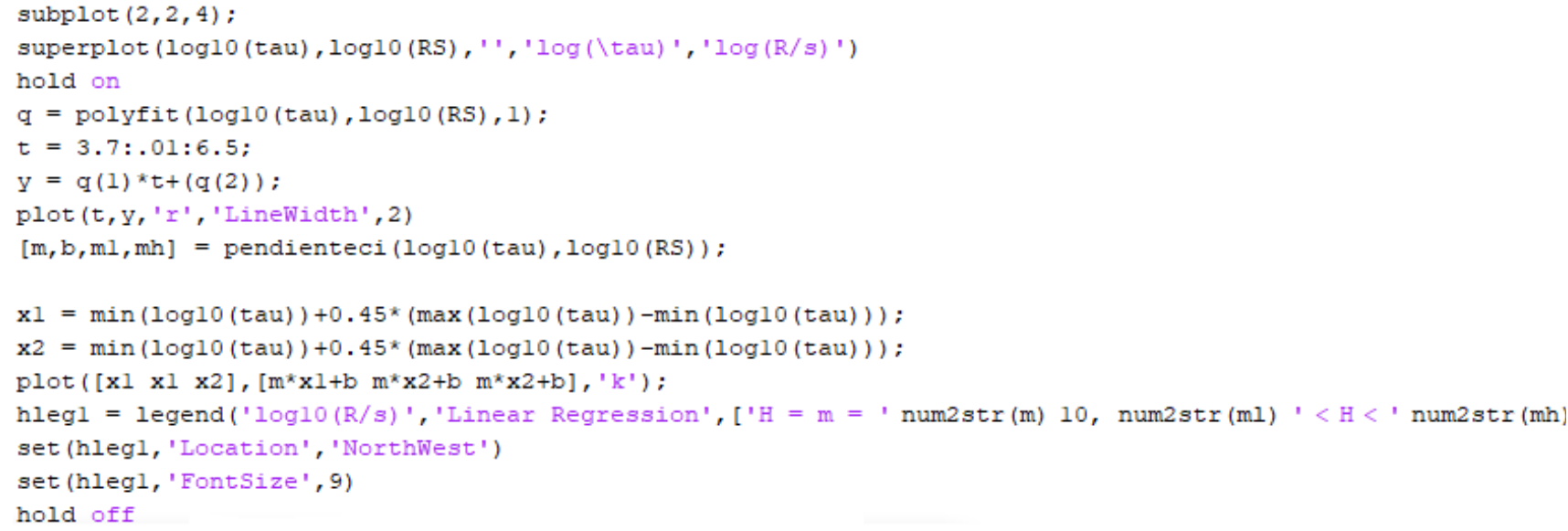

Figure 13: Calculation of the $H$ Parameter.

The test of the code ends by generating the graph shown in Figure 14, obtaining a value of $\mathrm{H}=0.80969$. 


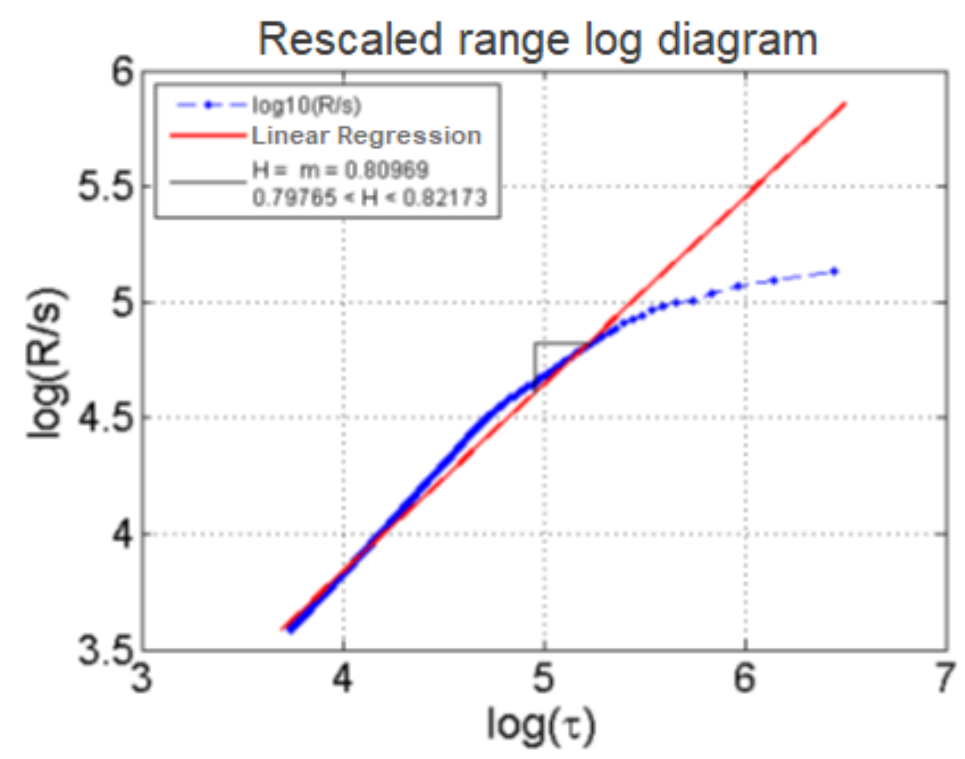

Figure 14: Result of Applying the Rescaled Range Process to the Data-Trace.

\section{DATA TRACE ANALYSIS}

Table 1 shows a summary of the tests performed on various images, thus having data-trace sizes of different lengths. For each case, the value of $\mathrm{H}$ and its respective variance is shown.

Table 1: Image Creation Time as a Function of Distance

\begin{tabular}{|l|c|c|c|}
\hline \multirow{2}{*}{$\begin{array}{c}\text { Image } \\
\text { (No. of Data) }\end{array}$} & Variance-time & Wavelet & R/S \\
\cline { 2 - 4 } IMG(7).jpg (131.228) & 0.5452 & 0.89891 & 0.6318 \\
& $(0.52508-0.56537)$ & $(0.79058-1.0072)$ & $(0.62559-0.63802)$ \\
\hline \multirow{2}{*}{ IMG(11).jpg (306.600) } & 0.8214 & 0.84313 & 0.82617 \\
& $(0.81705-0.82572)$ & $(0.7609-0.92536)$ & $(0.81947-0.83286)$ \\
\hline IMG(12).jpg (151.380) & 0.9545 & 0.88892 & 0.90861 \\
& $(0.95276-0.95632)$ & $(0.79577-0.98207)$ & $(0.90656-0.91067)$ \\
\hline \multirow{2}{*}{ IMG(17).jpg (921.600) } & 0.7725 & 0.83655 & 0.88424 \\
& $(0.76859-0.7765)$ & $(0.736-0.93711)$ & $(0.87676-0.89172)$ \\
\hline \multirow{2}{*}{ IMG(19).jpg (317.112) } & 0.7680 & 0.8722 & 0.77628 \\
& $(0.76093-0.77505)$ & $(0.78016-0.96424)$ & $(0.77324-0.77932)$ \\
\hline \multirow{2}{*}{ IMG(24).jpg (842.700) } & 0.8454 & 0.85291 & 0.85076 \\
& $(0.84121-0.84953)$ & $(0.74295-0.96286)$ & $(0.84352-0.858)$ \\
\hline \multirow{2}{*}{ IMG(26).jpg (1.710.000) } & 0.5230 & 0.82392 & 0.75045 \\
& $(0.51243-0.53361)$ & $(0.76072-0.88712)$ & $(0.74666-0.75424)$ \\
\hline \multirow{2}{*}{ IMG(28).jpg (2.764.800) } & 0.7730 & 0.81829 & 0.80969 \\
& $(0.76927-0.77681)$ & $(0.76886-0.86772)$ & $(.079765-0.82173)$ \\
\hline \multirow{2}{*}{ IMG(29).jpg (2.359.296) } & $(0.82067-0.82566)$ & $(0.76846-0.89347)$ & $(0.76644-0.77484)$ \\
\hline IMG(-2).jpg (3.147.264) & 0.8538 & 0.88095 & 0.77064 \\
& $(0.85073-0.85681)$ & $(0.8298-0.93392)$ & $(0.91845-0.9303)$ \\
\hline
\end{tabular}

For the issue of accuracy and bias of the different methods, there are detailed comparative studies, including the work done by [15], which details several results that show the advantage of Wavelet-based methods concerning these topics.

Figure 15 below summarizes the comparative tables extracted from the work of [15], which contain the results of the accuracy and bias analysis for the different methods addressed in the study. 


\begin{tabular}{|l|c|c|c|c|}
\hline \multirow{2}{*}{ Methods } & \multicolumn{3}{|c|}{ Mean Values of Estimated $H$ and $\Delta H$} \\
\cline { 2 - 5 } & \multicolumn{2}{|c|}{.6} & \multicolumn{2}{c|}{.7} \\
\cline { 2 - 5 } & $\hat{H}$ & $\Delta H(\%)$ & $\hat{H}$ & $\Delta H(\%)$ \\
\hline \hline Wavelet-based & .6002 & +0.026 & .7006 & +0.092 \\
& $(.573, .628)$ & & $(.673, .728)$ & \\
\hline Whittle's MLE & .6003 & +0.043 & .7003 & +0.044 \\
& $(.591, .610)$ & & $(.691, .710)$ & \\
\hline Periodogram & .6008 & +0.128 & .7025 & +0.358 \\
& $(.599, .603)$ & & $(.700, .705)$ & \\
\hline R/S-statistic & .6277 & +4.623 & .7118 & +1.689 \\
& $(.624, .632)$ & & $(.708, .716)$ & \\
\hline Variance-time & .5964 & -0.608 & .6917 & -1.192 \\
& $(.594, .599)$ & & $(.689, .695)$ & \\
\hline IDC $(t)$ & .5968 & -0.528 & .6919 & -1.153 \\
& $(.594, .600)$ & & $(.689, .695)$ & \\
\hline
\end{tabular}

\begin{tabular}{|l|c|c|c|c|}
\hline \multirow{2}{*}{ Methods } & \multicolumn{3}{|c|}{ Mean Values of Estimated $H$ and $\Delta H$} \\
\cline { 2 - 5 } & \multicolumn{2}{|c|}{.8} & \multicolumn{2}{c|}{.9} \\
\cline { 2 - 5 } & $\hat{H}$ & $\Delta H(\%)$ & $\hat{H}$ & $\Delta H(\%)$ \\
\hline \hline Wavelet-based & .8009 & +0.110 & .9010 & +0.108 \\
& $(.773, .828)$ & & $(.874, .929)$ & \\
\hline Whittle's MLE & .8004 & +0.044 & .9004 & +0.043 \\
& $(.791, .810)$ & & $(.891, .909)$ & \\
\hline Periodogram & .8040 & +0.496 & .9054 & +0.598 \\
& $(.802, .806)$ & & $(.903, .908)$ & \\
\hline R/S-statistic & .7916 & -1.053 & .8621 & -4.210 \\
& $(.787, .796)$ & & $(.857, .867)$ & \\
\hline Variance-time & .7814 & -2.327 & .8600 & -4.439 \\
& $(.778, .785)$ & & $(.856, .864)$ & \\
\hline IDC $(t)$ & .7812 & -2.349 & .8593 & -4.520 \\
& $(.778, .785)$ & & $(.855, .863)$ & \\
\hline
\end{tabular}

Figure 15: Results of the Accuracy and Bias Analysis for the Different Methods Performed by [15].

These tables show the relative error of the H estimates, of the different methods, taking as a base a standard sequence of 215 elements, generated by the Durbin-Levinson algorithm, which allows obtaining sequences with very precise values of $\mathrm{H}$, in this case, sequences with $\mathrm{H}=0.6 ; 0.7 ; 0.8$ and 0.9 respectively were used, obtaining the values shown above.

In the paper of [15], it is indicated that the estimation methods based on Wavelets and the Whittle MLE estimator are substantially more precise than the other methods usually used for the estimation of $\mathrm{H}$, since a good estimator should not only produce values close to the real one but also the estimates should have small values of variance. A table extracted from that work, containing the variances of the estimation of $\mathrm{H}$, for each of the methods, is shown below in figure 16.

\begin{tabular}{|l|c|c|c|c|}
\hline \multirow{2}{*}{ Methods } & \multicolumn{4}{|c|}{ Variances of Estimated $H$} \\
\cline { 2 - 5 } & .6 & .7 & .8 & .9 \\
\hline \hline Wavelet-based & $1.892 \mathrm{e}-04$ & $1.922 \mathrm{e}-04$ & $1.951 \mathrm{e}-04$ & $1.985 \mathrm{e}-04$ \\
\hline Whittle's MLE & $1.093 \mathrm{e}-05$ & $1.145 \mathrm{e}-05$ & $1.179 \mathrm{e}-05$ & $1.217 \mathrm{e}-05$ \\
\hline Periodogram & $1.288 \mathrm{e}-04$ & $1.306 \mathrm{e}-04$ & $1.291 \mathrm{e}-04$ & $1.279 \mathrm{e}-04$ \\
\hline R/S-statistic & $3.971 \mathrm{e}-04$ & $4.724 \mathrm{e}-04$ & $5.400 \mathrm{e}-04$ & $5.669 \mathrm{e}-04$ \\
\hline Variance-time & $1.928 \mathrm{e}-04$ & $2.571 \mathrm{e}-04$ & $3.405 \mathrm{e}-04$ & $3.826 \mathrm{e}-04$ \\
\hline IDC $(t)$ & $2.227 \mathrm{e}-04$ & $2.812 \mathrm{e}-04$ & $3.613 \mathrm{e}-04$ & $3.947 \mathrm{e}-04$ \\
\hline
\end{tabular}

Figure 16: Variances in the Estimation of $H$ for Methods used in [15].

To generate the values in this table, 100 sequences were used for each value of $\mathrm{H}$. Here it can be seen that the variance of the Whittle and Periodogram estimators are lower than that of wavelets, which is consistent since the Whittle estimator, in turn, is based on the periodogram but in general, the variance of all three is much lower than that of the other methods.

\section{RESULTS ANALYSIS}

The runtime results for each method and each of the data traces used is shown in table 2.

Table 2. Running times of the developed algorithms 


\begin{tabular}{|l|c|c|c|}
\hline \multirow{2}{*}{ Image (No. of Data) } & \multicolumn{3}{|c|}{ Running time (s) } \\
\cline { 2 - 4 } & Variance-time & Wavelet & R/S \\
\hline IMG(7).jpg (131.228) & 0.839629 & 0.226700 & 0.762884 \\
\hline IMG(11).jpg (306.600) & 2.756220 & 0.271206 & 1.042579 \\
\hline IMG(12).jpg (151.380) & 1.006201 & 0.232913 & 0.849324 \\
\hline IMG(17).jpg (921.600) & 14.725937 & 0.443980 & 2.165143 \\
\hline IMG(19).jpg (317.112) & 2.898986 & 0.275346 & 1.039639 \\
\hline IMG(24).jpg (842.700) & 12.903071 & 0.435374 & 2.031720 \\
\hline IMG(26).jpg (1.710.000) & 38.421592 & 0.725372 & 3.607628 \\
\hline IMG(28).jpg (2.764.800) & 73.2011481 & 1.043706 & 5.485256 \\
\hline IMG(29).jpg (2.359.296) & 61.269146 & 1.014881 & 4.7594446 \\
\hline IMG(-2).jpg (3.147.264) & 89.147451 & 1.130897 & 6.249511 \\
\hline
\end{tabular}

When analyzing each of the methods used based on the results obtained, it can be seen that the power spectral density method of the Wavelet transform is computationally much more efficient since when applying the wavelet transform the processing is reduced from $\mathrm{N}$ data to $\log 2$ of this value making its treatment much simpler.

It can be said that the robustness of an algorithm must consider all possible facets of the problem to be solved when developing an algorithm should not escape any detail that causes improper functioning of the algorithm. If a robust algorithm is built, any unexpected turn of the problem will be controlled by the algorithm, that is, it must be flexible to changes.

With the previous definition when testing with an image with completely black background (IMG(0).jpg) or 80\% black background (IMG(20).jpg) the only algorithm that generated response was the power spectral density method of the Wavelet transform, the other two methods do not generate any kind of response, so you can think that this algorithm is robust against the others.

\section{CONCLUSIONS}

If a long-term memory measurement is required in a time series, the Hurst parameter or exponent is the appropriate option to use. This parameter refers to the speed at which it decreases the autocorrelation of the time series while the offset between pairs of values increases.

For a value of $\mathrm{H}$ that is between 0.5 and 1 the exponent points to a time series that has positive autocorrelation and that will have that tendency in the long term, which means that when a high value is presented in the series it will possibly be followed by another of high value and that, also, the values after a long time will have a tendency to be high.

When you have $\mathrm{H}$ values in the zone between 0 and 0.5 a time series with long term changes with high and low values in neighboring pairs are generated, this means that a high value will possibly be followed by a low value.

The various existing methods for estimating the value of the Hurst parameter have each their particularity, some having very efficient response speeds for large data traces as is the case with the power spectral density method of the Wavelet transform that handles low response times. Others have very low variance values as is the case of the Whittle estimator (not developed in this paper).

\section{ACKNOWLEDGMENTS}

The authors would like to thank the Universidad Distrital Francisco José de Caldas and the LASER research group that supported the development and testing of the project.

\section{REFERENCES}


1. Hurst, H. E. (1951). Long Term Storage Capacity of Reservoirs. Transactions of the American Society of Civil Engineers. 116:770-799.

2. Hurst,H. E., Black, R. P.\&Simaika,Y. M. (1965). Long-Term Storage: An Experimental Study. London: Constable, 1965.

3. Plazas-Nossa, L., Ávila, M. A. \&Moncada, G. (2014).Estimating Hurst Exponent and Fractal Dimension for UV-VIS Absorbance Time Series Analysis. Ciencia e Ingeniería Neogranadina. 24(2):133-143.

4. Racine, R. (2011). Estimating the Hurst Exponent. Retrieved from:https://mosaic.mpi-cbg.de/docs/Racine2011.pdf

5. Cevizci, I., Erol, M. \&Oktug,S. F. (2006). Analysis of Multi-Player Online Game Traffic Based on Self-similarity. In Proceedings of 5th ACM SIGCOMM workshop on Network and system support for games, New York, USA, 2006.

6. Kaplan, I. (2013). Estimating He Hetrieved from:http://bearcave.com/misl/misl_tech/wavelets/hurst/index.html\#EstimatingHurstWithRs

7. Domínguez, D. L., Ardila, E.\&Moreno,J. F. (2010). Metodología e interpretación del coeficiente de Hurst. Odeon. 5:265-290.

8. Jeong, H.-D. J., McNickle, D. \&Pawlikowski, K. (2001). Hurst Parameter Estimation Techniques: A Critical Review. In Proceedings of ORSNZ, Conference Twenty Naught One, Christchurch, New Zealand, 2001, pp. 165-172.

9. Stan, C., Cristescu, C. M.\&Cristescu, C. P. (2014).Computation of Hurst Exponent of Time Series Using Delayed (Log-) Returns. Application to Estimating the Financial Volatility. Scientific Bulletin, Series A: Applied Mathematics and Physics. 76(3).

10. Feder, J. (1988).Fractals. Physics of Solids and Liquids, Boston, MA, USA: Springer,. 1988.

11. Millen, S. \&Beard, R. (2003). Estimation of the Hurst Exponent for the Burdekin River using the Hurst-Mandelbrot Rescaled Range Statistic. In First Queensland Statistics Conference, The University of Southern Queensland, Toowoomba, 1-3 October, 2003.

12. Navarro, X., Porée, F., Beuchée, A. \&Carrault, G. (2013). Performance analysis of Hurst exponent estimators using surrogatedata and fractional lognormal noise models: Application to breathing signals from preterm infants. Digital Signal Processing. 23(5):1610-16919.

13. López-Lambraño, A., Carrillo-Yee, E., Fuentes, C., López-Ramos, A. \& López-Lambraño, M. (2017). Una revisión de los métodos para estimar el exponente de Hurst y la dimensión fractal en series de precipitación y temperatura. Revista Mexicana de Física, 63(3):244-267.

14. Alvarez-Ramirez, J., Echeverria, J. C. \&Rodriguez, E. (2008). Performance of a high-dimensional R/S method for Hurst exponent estimation. Physica A: Statistical Mechanics and its Applications. 387(26):6452-6462.

15. Jeong, H.-D. J. (2002): Modelling of Self-Similar Teletraffic for Simulation. Ph.D. Thesis, University of Canterbury, July 2002. 
